\section{POS0211 PREDICTORS OF PROGRESSION AND MORTALITY IN PATIENTS WITH PREVALENT RHEUMATOID ARTHRITIS AND INTERSTITIAL LUNG DISEASE: A PROSPECTIVE COHORT STUDY}

N. Mena-Vázquez ${ }^{1}$, M. Rojas-Giménez ${ }^{2}$, C. M. Romero-Barco ${ }^{3}$, S. Manrique Arija ${ }^{1}$, F. Espildora ${ }^{4}$, M. C. Aguilar-Hurtado ${ }^{5}$, R. Ortega Castro ${ }^{6}$, I. Añón Oñate ${ }^{7}$, L. Pérez Albaladejo $^{8}$, F. Godoy-Navarrete ${ }^{1}$, I. Ureña ${ }^{1}$, M. Velloso Feijoo ${ }^{9}$, R. Redondo ${ }^{1}$, F. G. Jiménez-Núñez ${ }^{1}$, B. Panero Lamothe ${ }^{10}$, M. I. Padin-Martín ${ }^{5}$, A. FernandezNebro ${ }^{1}{ }^{1}$ Instituto de Investigación Biomédica de Málaga (IBIMA), Hospital Regional Universitario de Málaga (HRUM), Reumatología, Málaga, Spain; ${ }^{2}$ Instituto Maimónides de Investigación Biomédica de Córdoba (IMIBIC, UGC de Reumatología, Hospital Universitario Reina Sofía de Córdoba, Reumatología, 14004, Spain; ${ }^{3}$ Instituto de Investigación Biomédica de Málaga (IBIMA), Hospital Clínico Universitario Virgen de la Victoria, Reumatología, Málaga, Spain; ${ }^{4}$ Hospital Regional Universitario de Málaga (HRUM), Neumología, Málaga, Spain; ${ }^{5}$ Hospital Regional Universitario de Málaga (HRUM), Radiología, Málaga, Spain; ${ }^{6}$ Instituto Maimónides de Investigación Biomédica de Córdoba (IMIBIC). UGC de Reumatología, Hospital Universitario Reina Sofía de Córdoba., Reumatología, Córdoba, Spain; ${ }^{7}$ Hospital Universitario de Jaén, Reumatología, Jaén, Spain; ${ }^{8}$ Hospital Universitario Virgen de las Nieves, Reumatología, Granada, Spain; ${ }^{9}$ Hospital Universitario Virgen de Valme, Reumatología, Málaga, Spain; ${ }^{10}$ Hospital Clínico Universitario Virgen de la Victoria, Reumatología, Málaga, Spain

Objectives: To analyze the effect of disease-modifying antirheumatic drugs (DMARDs) and identify risk factors associated with disease progression and mortality in patients with rheumatoid arthritis associated with interstitial lung disease (RA-ILD). Methods: We performed a multicenter, prospective, observational study of patients with RA-ILD receiving DMARDs between 2015 and 2020. The patients were assessed using high-resolution computed tomography and pulmonary function tests at baseline and at 60 months. The main outcome measure at 60 months was worsening of FVC $>10 \%$ or DLCO $>15 \%$ and radiological progression or death. We recorded demographic and clinical characteristics, lung function, and the incidence of adverse events. A Cox regression analysis was performed to identify factors associated with worsening of ILD. Results: After 60 months, lung disease had stabilized in 66 patients $(56.9 \%)$, improved in $9(7.8 \%)$, and worsened in 23 (19.8\%). Eighteen patients $(15.5 \%)$ died, with a mean survival of 71.8 (1.9) months. Baseline characteristics of 116 with RA-ILD treated with DMARDs is in table 1.The Cox multivariate analysis revealed the independent predictors of worsening of RA-ILD to be usual interstitial pneumonia ( $\mathrm{HR}, 2.6[95 \% \mathrm{Cl}, 1.0-6.7])$, forced vital capacity (\%) (HR, 3.8 $[95 \% \mathrm{Cl}, 1.5-6.7])$, anticitrullinated protein antibody titers $(\mathrm{HR}, 2.8[95 \% \mathrm{Cl}, 1.1-$ $6.8]$ ), smoking ( $\mathrm{HR}, 2.5[95 \% \mathrm{Cl}, 1.1-6.2])$, and treatment with abatacept, tocilizumab, or rituximab (HR, $0.4[95 \% \mathrm{Cl}, 0.2-0.8]$ ). During follow-up, 79 patients $(68 \%)$ experienced an adverse event, mostly infection $(61 \%)$.

Conclusion: Lung function is stable in most patients with RA-ILD receiving treatment with DMARDs, although one third of patients die. Identifying factors of worsening in RA-ILD is important for clinical management.

\section{Table 1. Baseline characteristics of 116 with RA-ILD treated with DMARDs}

\begin{tabular}{|c|c|}
\hline Variable & Total $=116$ \\
\hline 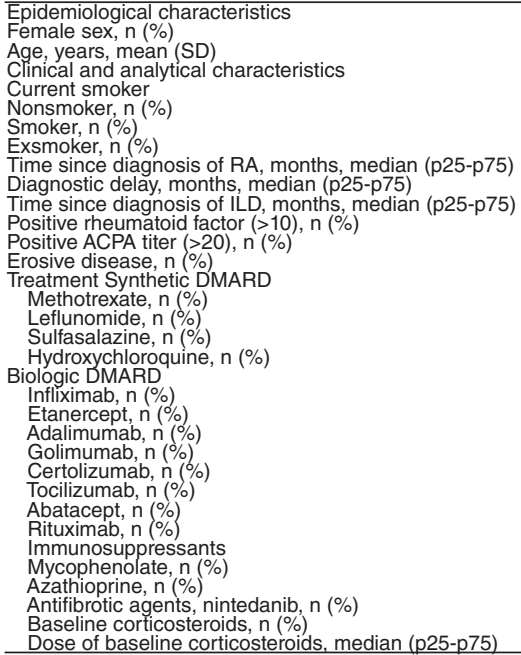 & $\begin{array}{l}57(49.1) \\
23(19.8) \\
36(31.0) \\
148.5(71.5-217.8) \\
8.5(4.9-16.8) \\
27.5(9.8-60.0) \\
111(95.7) \\
100(86.2) \\
76(65.5) \\
100(86.2) \\
51(44.0) \\
30(25.9) \\
9(7.8) \\
21(18.1) \\
50(43.1) \\
1(0.9) \\
6(5.2) \\
3(2.6) \\
3(2.6) \\
3(2.6) \\
6(5.2) \\
15(12.9) \\
13(11.2) \\
11(9.5) \\
7(6.0) \\
4(3.4) \\
1(0.9) \\
69(60.0) \\
5.0(0.0-7.5)\end{array}$ \\
\hline
\end{tabular}

Abbreviations. RA: rheumatoid arthritis; ILD: interstitial lung disease; ACPA: anticyclic citrullinated protein antibody; DMARD: disease-modifying antirheumatic drug; SD: standard deviation.

Acknowledgements: Grant for Medical Researchers of the "Fundación Española de Reumatología" 2019. declare.

Disclosure of Interests: None declared

DOI: 10.1136/annrheumdis-2021-eular.1090

\section{POS0212 COMPARISON OF PATIENT CHARACTERISTICS ANDTREATMENT PATTERNS ACROSS BODY MASS INDEX CATEGORIES IN PATIENTS WITH PSORIATIC ARTHRITIS AND RHEUMATOID ARTHRITIS}

E. Vallejo-Yagüe ${ }^{1}$, T. Burkard ${ }^{1}$, B. Moeller ${ }^{2}$, A. Finckh ${ }^{3}$, A. M. Burden ${ }^{1} .{ }^{1}$ ETH Zurich, Department of Chemistry and Applied Biosciences, Institute of Pharmaceutical Sciences, Zurich, Switzerland; ${ }^{2}$ Inselspital, University Hospital of Bern, Rheumatology, Immunology and Allergy, Bern, Switzerland; ${ }^{3}$ University Hospitals Geneva, Division of Rheumatology, Geneva, Switzerland

Background: Higher prevalence of obesity has been observed in psoriatic arthritis (PsA) and rheumatoid arthritis (RA) versus the general population, and abnormal body mass index has been associated with worse rheumatic markers. Objectives: To describe PsA and RA patients in Switzerland, stratified by body mass index (BMI) category.

Methods: We performed a descriptive cohort study in PsA and RA patients registered in the Swiss Clinical Quality Management in Rheumatic Diseases (SCQM) database. Two distinct cohorts were generated based on patient diagnosis (PsA or RA) and analysed separately but using similar approaches. In both cohorts, we included patients treated for the first time with biologics or targeted synthetic disease-modifying anti-rheumatic drugs (tsDMARDs), and considered the treatment start as index date. Patients without baseline $\mathrm{BMI}$ were excluded. Patients were stratified by BMl category at the start of biologic/tsDMARD treatment, defined as underweight (BMI<18.5), normal weight (BMl 18.5-24.9), overweight (BMI 25.0-29.9), and obese (BMI $\geq 30$ ). In the PsA cohort, underweight and normal weight groups were merged due to low numbers. The proportion of patients categorized as overweight or underweight were compared to national statistics from the Swiss Federal Statistical Office. Information on patient demographics (e.g., age, sex BMI, life-habits), disease-specific characteristics (e.g., disease activity scores, health questionnaires, biomarkers), co-medications and comorbidities were summarized at the start of the first biologic/tsDMARD treatment. Patient characteristics across BMI categories were compared, using the normal weight category as reference group. Additionally, we summarized the frequency and reasons for recorded treatment stop/switch at $\leq 6$ months, 6 to 12 months, and >12months from treatment start, and illustrated the prescription patterns for first and second biologic/tsDMARD treatment, stratifying by BMI. Results: We identified 819 PsA [39.7\% normal weight, 36.5\% overweight, $23.8 \%$ obese] and 3217 RA patients [ $4.4 \%$ underweight, $46.8 \%$ normal weight, $31.8 \%$ overweight, $17.0 \%$ obese]. Figure 1 illustrates the prevalence of overweight and obesity in each cohort stratified by sex, compared to the national average. When comparing obese patients to those with normal weight, both PsA and RA obese patients had significantly higher $\mathrm{C}$-reactive protein, worse disease activity score, lower quality of life (QoL) measures, and more frequent cardiovascular disease and diabetes. Among PsA patients, the overweight and obese had worse physician-assessed skin manifestation and patient-reported pain compared to the normal weight group. While in RA, the obese patients had higher erythrocyte sedimentation rate, smaller prevalence of seropositive patients, lower frequency of fractures/surgeries, and higher tender joint counts, but similar swollen joint counts, when compared to the normal weight group. Adalimumab and etanercept, were the most commonly prescribed drugs as first biologic/tsDMARD treatment in both PsA and RA cohorts and among every BMI category. Overall, 55\% PsA and 56\% RA patients had recorded treatment stop/switch. Among RA patients, significantly fewer obese patients reported treatment stop/ switch at >12 months from treatment start, compared to the normal weight group. Adalimumab and etanercept were also the most commonly prescribed second biologic/tsDMARD treatment, but for the obese group among PsA patients (adalimumab, golimumab) and the obese group in the RA cohort (adalimumab, rituximab). Conclusion: In this national wide study, we observed that the prevalence of obesity in RA and PsA was higher than that of the general Swiss population. Obese PsA/RA patients starting first biologic/tsDMARD treatment presented worse disease activity and poorer QoL than normal weight patients. Results suggest to take BMI into consideration when treating PsA and RA patients.

Overweight and obese prevalence

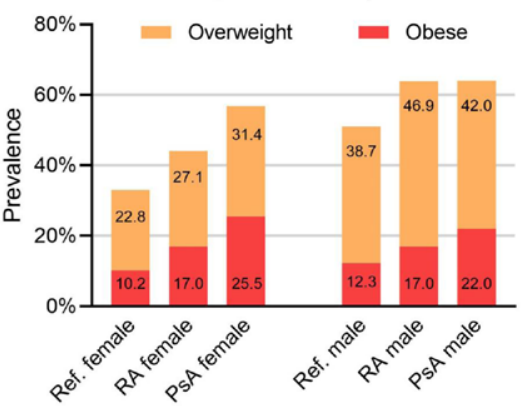

Ref. Reference: $>15$ years old Swiss population in 2017 sbefragung 2017 - Korrigierte Version 25.09 .2020

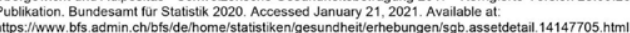
RA rheumatoid arthritis. Study dats. PSA psoriatic arthrits Study dot 
Acknowledgements: We would like to thank all patients and rheumatologists contributing to the SCQM registry, as well as the entire SCQM staff. A list of rheumatology offices and hospitals which contribute to the SCQM registry can be found at http://www.scqm.ch/institutions. The SCQM is financially supported by pharmaceutical industries and donors. A list of financial supporters can be found at http://www.scqm.ch/sponsors.

Disclosure of Interests: Enriqueta Vallejo-Yagüe: None declared, Theresa Burkard: None declared, Burkhard Moeller Speakers bureau: AbbVie, Bristol Myers, Eli Lilly, Janssen, Pfizer, Roche, Novartis, Merck, Axel Finckh Speakers bureau: Pfizer, Eli-Lilly, Paid instructor for: Pfizer, Eli-Lilly, Consultant of: AbbVie, AB2Bio, BMS, Gilead, Pfizer, Viatris, Grant/research support from: Pfizer, BMS, Novartis, Andrea Michelle Burden: None declared

DOI: 10.1136/annrheumdis-2021-eular.1348

\section{POS0213 20 YEAR FOLLOW-UP OF CARDIOVASCULAR EVENT RISK IN RHEUMATOID ARTHRITIS COMPARED TO DIABETES}

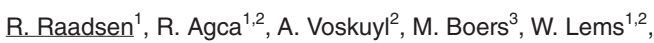

M. Nurmohamed ${ }^{1,2} .{ }^{1}$ Amsterdam Rheumatology and Immunology Center, location Reade, Rheumatology, Amsterdam, Netherlands; ${ }^{2}$ Amsterdam Rheumatology and Immunology Center, location VU University Medical Center, Rheumatology, Amsterdam, Netherlands; ${ }^{3}$ Amsterdam University Medical Center, Location VU University Medical Center, Biostatistics and Epidemiology, Amsterdam, Netherlands

Background: Patients with rheumatoid arthritis have an increased risk for developing cardiovascular diseases (CVD) compared to the general population, similar to the CVD risk in patients with diabetes mellitus. However, there are no controlled studies investigating the incidence of cardiovascular (CV) events in RA patients with follow up of more than 20 years.

Objectives: The objectives of the current study were to investigate the incidence rates of CV events in a long-term follow up cohort of RA patients, and to compare these to a similar cohort representing the general population, ie. The Hoorn study.

Methods: The CARRÉ study is an ongoing prospective cohort study, which started in 2001, investigating CV mortality and morbidity in 353 randomly selected patients with RA. Primary endpoints, i.e. verified medical history of coronary, cerebral or peripheral arterial disease, were determined at baseline, and after three, ten, fifteen and twenty years of follow up. Patients were censored at the date of an experienced CV event or their death. Incidence density rates per 100 patient years were calculated. Data were compared to results from the Hoorn study, a Dutch cohort study of glucose metabolism and other CV risk factors that began in 1989. All 2,484 participants were subject to an extensive and repeated CV screening program similar to that used in the CARRÉ study.

Results: After 20 years of follow up 118 patients (33\%) developed at least one CV event in the Carré group. Mean (SD) follow up time was 11 (6) years with a total of 3,500 years at risk and an incidence rate of 3.4 per 100 patient-years; this is slightly up from the figure reported at 15 years, i.e. 3.2 per 100 patient-years. A CV event-free survival curve is shown in figure 1. After 30 years of follow up, 295 participants of the Hoorn study had developed a CV event, during a mean follow up time 20 (8) years. Total time at risk was 50,000 years, with an incidence rate of $0.6 \mathrm{CV}$ events per 100 patient years.

Conclusion: In our cohort the incidence rate of $\mathrm{CV}$ events in RA patients has remained consistently high when compared with the general population, despite better control of RA inflammation in recent years. This again confirms the need for timely CVD-risk screening and management.

REFERENCES:

[1] Agca R, Hopman L, Laan KJC, van Halm VP, Peters MJL, Smulders YM, et al. Cardiovascular Event Risk in Rheumatoid Arthritis Compared with Type 2 Diabetes: A 15-year Longitudinal Study. J Rheumatol. 2020;47(3):316-24.

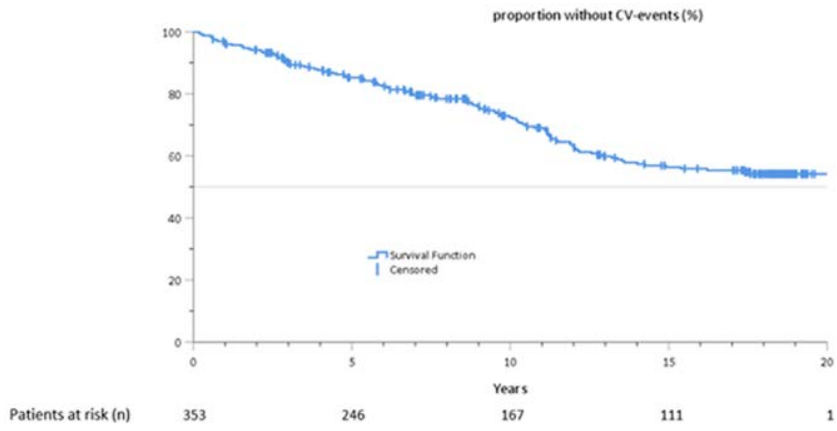

Figure 1. Survival curve of participants with rheumatoid arthritis. $\mathrm{RA}=$ rheumatoid arthritis
Disclosure of Interests: None declared

DOI: 10.1136/annrheumdis-2021-eular.1744

\section{POS0214 \\ ASSOCIATION BETWEEN C-REACTIVE PROTEIN AND 10-YEAR RISK OF CARDIOVASCULAR DISEASE IN RHEUMATOID ARTHRITIS USING THE ERS-RA SCORE: A CROSS-SECTIONAL ANALYSIS OF THE CORDIS COHORT}

G. L. Erre ${ }^{1}$, F. Cacciapaglia ${ }^{2}$, G. Sakellariou ${ }^{3}$, A. Manfredi ${ }^{4}$, E. Bartoloni Bocci $^{5}$, O. Viapiana ${ }^{6}$, M. Fornaro ${ }^{2}$, M. Dessi ${ }^{7}$, A. A. Mangoni ${ }^{8}$, B. L. Palermo ${ }^{9}$, E. Gremese ${ }^{10}$, G. Cafaro ${ }^{5}$, V. Nucera ${ }^{11}$, C. Vacchi ${ }^{12}$, F. R. Spinelli ${ }^{13}$, F. Atzeni ${ }^{11}$ M. Piga ${ }^{14}$ on behalf of "Cardiovascular Obesity and Rheumatic Disease Study (CORDIS)" Group of the Italian Society of Rheumatology (SIR).. 'University and AOU of Sassari, Department of Medical, Surgical and Experimental Sciences, Sassari, Italy; ${ }^{2}$ University and AOU, Policlinico of Bari, Department of Emergency and Organs Transplantation, Bari, Italy; ${ }^{3}$ University of Pavia, Istituti Clinici Scientifici Maugeri IRCCS Pavia, Pavia, Italy; ${ }^{4}$ Azienda Ospedaliera Universitaria Policlinico di Modena, Unit of Rheumatology, Modena, Italy; ${ }^{5}$ University of Perugia, Department of Medicine and Surgery, Perugia, Italy; ${ }^{6}$ University of Verona, Dipartimento di Medicina, Verona, Italy; ${ }^{7}$ University of Cagliari, Department of Medical Sciences and Public Health, Cagliari, Italy; ${ }^{8}$ Flinders University, College of Medicine and Public Health, Adelaide, Australia; ${ }^{9}$ University of Pavia, IRCSS San Matteo, Pavia, Italy; ${ }^{10}$ Policlinico Gemelli, Università Cattolica del Sacro Cuore, Dipartimento di Scienze Mediche e Chirurgiche, Roma, Italy; ${ }^{11}$ University of Messina, Dipartimento di Medicina Clinica e Sperimentale, Messina, Italy; ${ }^{12}$ University of Modena and Reggio Emilia, Clinical and Experimental Medicine PhD Program, Modena, Italy; ${ }^{13}$ Università La Sapienza, Dipartimento di Scienze Cliniche Internistiche, Anestesiologiche e Cardiovascolari, Roma, Italy; ${ }^{14}$ University of Cagliari, Department of Medical Sciences and Public Health, Monserrato, Italy

Background: Rheumatoid arthritis (RA) is associated with an increased risk of atherosclerotic cardiovascular disease (CVD). The Expanded Cardiovascular Risk Prediction Score for Rheumatoid Arthritis (ERS-RA) estimates the 10-year risk of myocardial infarction, stroke or CVD-related death based on conventional and RA-specific (clinical disease activity index, CDAl, disease duration, glucocorticoid use) risk factors (1).

Objectives: We evaluated the associations between ERS-RA 10-year risk of CVD, high-sensitivity C-reactive protein (hs-CRP) concentrations, and pharmacological treatment in 1,251 RA patients collected by the "Cardiovascular Obesity and Rheumatic Disease Study (CORDIS)" group of the Italian Society of Rheumatology (SIR)

Methods: We assessed independent associations between ERS-RA risk score and each relevant variable using multivariate regression (ENTER approach; listwise deletion analysis). Given the relatively high number of missing hs-CRP data $(n=385)$, regression analysis was also performed using multiple imputation (10 sets, Stata 16.1). Regression models were not adjusted for independent variables included in the ERS-RA score.

Results: Among 1,251 RA patients [mean (SD) age 60.4(9.3), range (40 80 ) years; $78 \%$ female; mean (SD) disease duration, 11.6(8) years; mean (SD) CDAI, 9(9); mean (SD) HAQ, 0.77(0.7); mean (SD) hs-CRP, 6.8(12) $\mathrm{mg} / \mathrm{L}$ ] the estimated 10-year CVD risk was $11.6(0.9) \%$ [mean (SD)]. Regarding treatment, 539(43\%) received glucocorticoids, 676(54\%) a biological or targeted synthetic disease-modifying anti-rheumatic drug (b/tsDMARD) (n missing=1), and $885(81 \%$ ) at least one conventional synthetic DMARD (csDMARD). Ninety-three (7.4\%) patients did not receive any treatment. After adjusting for the use of b/tsDMARD and csDMARD, hs-CRP concentrations were significantly associated with 10-year risk of CVD both in standard multiple regression ( $n=865$; coefficient $=0.005$ for each $10 \mathrm{mg} / \mathrm{L}$ hs-CRP increment, $95 \%$ confidence interval (0.000-0.100), $p=0.043$ ) and after multiple imputation $(n=1,251$; coefficient $=0.005$ for each $10 \mathrm{mg} / \mathrm{L}$ hs-CRP increment, $95 \%$ confidence interval $(0.000-0.114), p=0.035)$ (Table 1). This corresponds to an increase of 10 -year CV risk of $1 \%$ for every $20 \mathrm{mg} / \mathrm{L}$ increase in hs-CRP concentrations.

Conclusion: In a large cohort of RA patients, we observed a significant, positive, and independent association between hs-CRP concentrations and 10-year CV risk estimated by ERS-RA. The cross-sectional design of the study did not allow to establish a cause-effect relationship between hs-CRP and CV risk. Given that conventional $\mathrm{CV}$ risk factors and inflammation-related variables are accounted for in the ERS-RA risk score, other, unexplored, mechanisms may underlie the observed association between hs-CRP and CV risk.

\section{REFERENCES:}

[1] Solomon, D. H., et al. "Derivation and internal validation of an expanded cardiovascular risk prediction score for rheumatoid arthritis: a Consortium of Rheumatology Researchers of North America Registry Study." Arthritis \& rheumatology 67.8 (2015): 1995-2003. 